\title{
Procedure Link Group
}

National Cancer Institute

\section{Source}

National Cancer Institute. Procedure Link Group. NCI Thesaurus. Code 1117510.

A sequence of characters used to link multiple procedures to a single finding. 